\title{
Cervicocephalic Arterial Dissection
}

\section{Marzie Abotorabi}

Shahid Sadoghi University of Medical Sciences, Yazd, Iran.

\begin{abstract}
Cervicocephalic arterial dissection is not a common disease (2.5\% of total brain infarction) and its pathogenesis is still not fully understood. However, it seems that it is multifactorial And rarely seen in the postpartum priod.

A 40-year-old female patient who referred to emergency department because she experienced right lower limb Paresis 10 days following the delivery and her Paresis was progressed within 72 hours. She has no history of DM ,HTN, preeclampsia and underlying cerebrovascular disease

On evaluations, Brain M RI revealed multiple infarction in the left M CA territory . ECG, TTE ,coagulation and rheumatologic tests were normal. leftextracranial carotid artery dissection was found in the cervical M RA.

The likelihood of postpartum dissection is rare , But it could be due to vasculardamage associated with the Valsalva maneuver during labor ; hemodynamic and hormonal changes due to pregnancyare also involved.
\end{abstract}

Key words: postpartum priod, Cervicocephalic arterial dissection, ischemic stroke 